\title{
LA REFORMA DEL SENADO ITALIANO: LUCES Y SOMBRAS*
}

\author{
TOMMASO EDOARDO FROSINI \\ Catedratico de Derecho Publico Comparado \\ Universidad de Sassari
}

1. El 16 de noviembre de 2005 el Parlamento italiano ha aprobado, según el procedimiento previsto por el artículo 138 del texto constitucional, una modificación importante que afecta a la segunda parte de la Constitución. Sin embargo, para que la reforma sea definitiva - teniendo en cuenta que ya ha sido aprobada por la mayoría absoluta de las Cámaras - y se convierta en una nueva norma constitucional, es necesario el voto favorable del cuerpo electoral expresado mediante referéndum ${ }^{1}$. Entre las más significativas novedades de la reforma constitucional se encuentra la modificación del Senado, que pasaría a convertirse en "federal" y, por tanto, representativo de las Regiones. En relación con las que pueden ser las reservas críticas al nuevo Senado federal, que examinaremos en este escrito, debemos advertir sin embargo que emergen también algunos aspectos positivos, en particular uno, se pone fin al bicameralismo perfecto y paritario. ¿No era este uno de los mayores problemas de la organización parlamentaria italiana? ¿Cuántas veces hemos leído, o escrito, que el bicameralismo italiano representa una anomalía en el panorama de los sistemas parlamentarios europeos, en los que las segundas Cámaras no son una réplica de las primeras, pero tienen, sin embargo, su función especí-

* Traducido del italiano por Yolanda Gómez Lugo (Universidad Carlos III).

1 Según el tenor literal del artículo 138 de la Constitución italiana: «Las leyes de revisión de la Constitución y demás leyes constitucionales serán adoptadas por cada una de las Cámaras en dos votaciones sucesivas con intervalo no menor de tres meses, y serán aprobadas por mayoría absoluta de los componentes de cada Cámara en la segunda votación. Dichas leyes serán sometidas a referéndum popular cuando, dentro de los tres meses siguientes a su publicación, lo solicite una quinta parte de los miembros de una Cámara o 500.000 (quinientos mil) electores o 5 (cinco) Consejos Regionales. La ley sometida a referéndum no se promulgará si no fuere aprobada por la mayoría de los votos válidos. No habrá lugar a referéndum si la ley hubiese sido aprobada en la segunda votación en cada una de las Cámaras por una mayoría de dos tercios de sus respectivos componentes." 
fica?² Y además, ¿̨cuántas veces hemos leído, y escrito, que el bicameralismo paritario ralentiza y debilita la actividad legislativa, reduciéndola a una navette tediosa donde termina prevaleciendo la mediación, los compromisos, con perjuicio de la eficacia y la claridad del acto legislativo, que debería ser general y abstracto? Aún más, ¿cuántas veces hemos leído, y escrito, que un sistema regional, a mayor razón si acentuado como el actual, necesita de una segunda Cámara privada de la relación fiduciaria con el Gobierno y representativa, en la medida de lo posible, de las autonomías territoriales? Sobre este punto, me gustaría traer a colación las palabras pronunciadas por Costantino Mortati ante la Asamblea Constituyente el 18 de septiembre de 1947: «La reforma regional no estaría completa, sino que se vería frustrada por los motivos y en los términos que han informado las instituciones, desviándose de la finalidad política que la ha promovido, si no encontrase su desarrollo y su situación en el ordenamiento del Parlamento[...] Donde la necesidad de dar a las regiones una voz específica en el Parlamento, de hacer [...] un centro unitario de intereses organizados para hacer valer unitariamente y de modo institucional ${ }^{3}$. Afirmaciones que medio siglo después, siguen siendo válidas, si no validísimas.

2. Efectivamente, del conjunto del proyecto de reforma constitucional votado por el Parlamento, lo concerniente a la modificación del bicameralismo, con la previsión del Senado federal de la República, es paradójicamente lo más auspiciado, pero al mismo tiempo lo más criticado. Adelantamos ya que la modificación del bicameralismo italiano puede no pasar necesariamente a través de la creación de una segunda Cámara como la alemana, esto es, el Bundesrat, compuesta por delegados designados (o revocados) por los gobiernos de los Laender. Incluso admitiendo que ésta sea la solución justa, debe afirmarse que el Senado italiano tiene su historia, su tradición constitucional, que no puede anularse radicalmente para convertirlo sólo en una sede de representación de los delegados regionales. Por tanto, sería oportuno para las regiones de carácter institucional, que el Senado, aún siendo "federal", no se convierta en un mero lugar de "inválidos de la Constitución,, por retomar la abandonada definición de Luigi Palma (que se remonta a 1869 y referida a la reforma del Senado de la época) ${ }^{4}$. Por tanto, desde el punto de vista de la salvaguardia de la tradición constitucional del Senado, y no solo, insisto, que el pro-

2 Cfr. L. PALADIN, Tipologia e fondamenti giustificativi del bicameralismo. Il caso italiano, en Quaderni costituzionali, n. 2, 1984; F. Rescigno, Disfunzioni e prospettive di riforma del bicameralismo italiano, Milano, Giuffrè, 1995.

3 Expresión tomada del volumen de N. Occhiocupo, La "Camera delle Regioni", Milano, Giuffrè, 1975 , p.58.

4 Véase, L. PALMA, La riforma del Senato in Italia, en Nuova Antologia, 1882 (ahora reeditado en el volumen de G. Spadolini, La riforma del Senato nell'Italia Unita. Fra Depretis e Giolitti, Firenze, Le Monnier, 1987, p.49 ss.); consúltese también las importantes consideraciones de Giorgio Arcoleo, constitucionalista, parlamentario y redactor en 1910 de un proyecto de reforma del Senado de la época: G. Arcoleo, Discorsi parlamentari, a cargo y con un prólogo de Tommaso Edoardo Frosini, (colección del Archivio storico del Senato della Repubblica), Bologna, il Mulino, 2005. 
yecto de reforma del mismo, al menos en la parte relativa a su composición, es quizá el menos traumático que se podía imaginar, pero al mismo tiempo no tiene en cuenta algunas exigencias de cambio. Más adelante veremos con detalle qué prevé el proyecto de reforma en relación a la composición y organización del Senado y por lo que respecta a los procedimientos legislativos. Sin embargo, previamente quisiera intentar ofrecer una respuesta a un interrogante, que se sitúa en el fundamento mismo del tema objeto de este escrito: ¿cuáles son, especialmente hoy, las razones que inducen a una reforma del bicameralismo, con la introducción de una segunda Cámara que sea lo más representativa posible de las autonomías territoriales, pero que no prescinda del todo de su función parlamentaria? Aquí me limitaré a ofrecer unas respuestas rápidas indicando al menos cuatro de los argumentos más significativos.

2.1. El primer argumento es el relativo a la reforma del Título Quinto de la Constitución, que tuvo lugar en 2001, con la repartición de las competencias legislativas entre el Estado y las Regiones y con la fijación de un amplio catálogo de materias competentes (consúltese el artículo 117 de la Constitución italiana); de ahí, la exigencia de situar formas de representación regionales en una de las dos Cámaras parlamentarias, para consentir la participación en la determinación de la potestad legislativa a favor de la periferia, y evitar la perpetuación de conflictos de atribución entre el Estado y las Regiones sobre la reivindicación de las competencias, que obliga a la Corte Constitucional a desarrollar un rol de suplente legislativo "no solicitado, ni bien acogido" $"$.

2.2. Además existe el argumento de la forma de gobierno; en efecto, solo superado el actual bicameralismo paritario es posible modificar (como parece, se pretende hacer) la forma de gobierno parlamentaria en el sentido del denominado premierato, que excluye al Senado del circuito fiduciario, quedando la Cámara de los diputados como única titular ${ }^{6}$. Apartando al Senado del poder fiduciario no solo se hace posible el premierato, sino que también se evita el peligro, como sucedió en 1994, de la formación de mayorías diferentes en las dos Cámaras.

2.3. El tercer argumento es el que tiene fuerza constitucional, por así decirlo. En efecto, la exigencia de transformar una de las dos Cámaras en una Cámara de las autonomías territoriales lo encontramos en una ley constitucional, la n. 3 del 2001, en su artículo 11. En éste se procede a reformar la Comisión bicameral para las cuestiones regionales, aumentando los poderes y previendo la inserción de representantes de las autonomías locales, pero "Hasta la revisión de las normas del Título I de la Constitución”. Se trata, pues, de una especie de "promesa constitucional", un empeño que el legislador constitucional ha toma-

5 Expresión empleada por Gustavo Zagrebelsky, como Presidente de la Corte Constitucional italiana, en la Conferencia de prensa anual que tuvo lugar en enero de 2004: La giustizia costituzionale nel 2003. Relazione del Presidente Gustavo Zagrebelsky, en Giurisprudenza costituzionale, n.5, 2004, pag. 3419 ss.

6 Sobre el premierato como forma de gobierno, véanse los trabajos recopilados en el volumen Il Premierato nei governi parlamentari,dirigido por T. E. Frosini, Torino, Giappichelli, 2004. 
do consigo mismo para reformar el actual bicameralismo paritario transformándolo en diferenciado.

2.4. El cuarto y último de los argumentos es el del federalismo, y se refiere al tipo de Estado que se quiere construir. No obstante, sobre este punto, se advierte que existe una incertidumbre generalizada sobre la calificación jurídica del federalismo, en cambio, existe una certeza constante sobre la organización del mismo, esto es, que todos los Estados más o menos federales o descentralizados internamente prevén una segunda Cámara representativa de las autonomías territoriales. A esta conclusión se llega fácilmente a través de la investigación del Derecho comparado 7 . En efecto, si se examina la estructura del bicameralismo en los Estados federales, se llega a la conclusión de que una de las dos Cámaras del Parlamento está destinada a facilitar la representación de los entes territoriales, o al menos de aquellos entes que tienen potestad legislativa, si bien, las diferencias se refieren a los criterios de elección de representantes y las competencias a éstos asignadas. Con este fin, conviene también puntualizar otro aspecto: una Cámara representativa de las autonomías territoriales se convierte en tal, no tanto y no sólo, por estar compuesta por representantes de los entes descentralizados, sino más bien por las funciones que ésta está llamada a desarrollar. La participación en el procedimiento de formación de algunas leyes, se convierte, efectivamente, en la actividad constitucionalmente más relevante de las Cámaras de las autonomías territoriales; además, se encuentran las actividades ejecutiva, de control y de designación. La participación en el procedimiento legislativo es el dato común a todas las Cámaras representativas de las autonomías territoriales; en cambio, no hay coincidencia por lo que se refiere a las formas de la representación. En efecto, a través de una panorámica del Derecho comparado, podemos destacar la presencia de al menos tres modos de representar. En primer lugar, aquellas en las que los representantes de los Estados coinciden con sus Ejecutivos, en cuanto son nombrados por éstos, revocados y seleccionados entre los miembros mismos de los Ejecutivos, siguiendo el modelo del Bundesrat alemán; aquella otra en que los representantes de los Estados son elegidos por sufragio universal por parte de la población de cada Estado, según el modelo de Senado estadounidense (tras la enmienda de 1913); y por último, aquellas en que los representantes del Estados son elegidos por los Parlamentos de los Estados miembros, pero no necesariamente pertenecen a los Parlamentos mismos, según el modelo del Bundesrat austriaco. Hay también un cuarto modo de representación de tipo mixto, elección y designación, que encontramos en España y Bélgica.

2.5. Del modo en que las autonomías territoriales entran a formar parte de la Cámara representativa deriva, pues, una serie de problemas que me limito aquí simplemente a señalar. Me refiero al problema del mandato imperativo: está claro que los miembros designados por los Gobiernos territoriales tienen

7 Cfr. T. E. FROSINI, Federalismo e bicameralismo, en el volumen. I percorsi del federalismo, dirigido por B.Caravita, Milano, Giuffrè, 2004, p.117 ss. 
un vínculo de mandato, es decir, están llamados a desarrollar su actividad según un mandato perfectamente precisado que les vincula al órgano que les ha designado; éstos no representan al territorio, sino más bien al Gobierno de los Estados miembro, que no es lo mismo ${ }^{8}$. Bien distinta es la situación de quienes son elegidos por el cuerpo electoral y, en parte, también quienes han sido designados por los Parlamentos de los Estados miembros; aquí el vínculo de mandato se debilita porque la elección postula una determinada forma de libertad de actuación política (al límite se puede prever el recall); éstos están llamados a responder de su actuación ante el electorado, o bien ante los Parlamentos que los han designado. El nexo de unión con el territorio existe, pero no es directo porque se fija mediante la elección. En el caso del sistema austriaco, la elección por parte de los Parlamentos de cada Estado hace emerger claramente las relaciones de fuerza partidista presente en el seno de las Asambleas legislativas de cada Land.

Por último, un dato relativo a la organización de las segundas Cámaras en los Estados federales es aquel sobre el número de representantes asegurados a cada entidad. Sobre ello la doctrina, sobre todo la alemana, ha elaborado una concepción basada en dos criterios: uno aritmético, según el cual cada ente estatal tiene un número igual de representantes, y otro geométrico, en virtud del cual el número de representantes varía en razón de la población y/o del territorio del Estado miembro. La cuestión no es baladí, y adopta un perfil de la relación entre federalismo y democracia. Está claro que allí donde se privilegia el criterio aritmético, se subraya mayormente una mayor expansión del principio federal; por el contrario, el criterio geométrico se justifica sobre el principio democrático, donde cada uno cuenta por lo que vale como ciudadano y como parte del territorio?.

3. A grandes rasgos, estos son algunos de los problemas relativos a una segunda Cámara representativa de las autonomías territoriales. Y son, además, los argumentos que apuestan por una modificación significativa del actual sistema bicameral italiano. Ahora bien, ¿son también los argumentos que se encuentran en el proyecto de reforma constitucional que ha sido aprobado por el Parlamento? Antes de enumerarlos y examinarlos, quisiera, sin embargo, aclarar una cuestión. El proceso de reforma ha producido ya un primer resultado que no puede descuidar, que es el de haber difundido y hecho adquirir conciencia de la necesidad e inderogabilidad de la reforma del Senado. Desde el punto de vista de la cultura parlamentaria, se ha entrado en un orden de ideas según el cual del modo en que está configurado el Senado, no lo estará más, porque cambiarán sus competencias y (probablemente) su representación. Tengo la impresión que este aspecto concretamente comienza a ser advertido sobre todo por los senadores, esto es, por aquellos que "naturalmente" estarían en situación

8 Sobre ello puede consultarse la reciente contribución de R. Scarciglia, Il divieto di mandato imperativo. Contributo a uno studio di diritto comparato, Padova, Cedam, 2005.

9 Véase la reconstrucción de F. PALERMO, Germania e Austria: modelli federali e bicamerali a confronto, Trento, Università degli studi di Trento, 1997. 
de transformar el Senado y, de este modo, ceder sus prerrogativas. Este me parece un paso adelante que, de cualquier modo, diluye la conocida paradoja de las reformas constitucionales ${ }^{10}$.

Es indudable que al cambio debe corresponder el logro de un nuevo orden bicameral que satisfaga las exigencias del sistema federal italiano. Es precisamente en este punto donde venimos a examinar qué prevé el proyecto de Senado federal de la República ${ }^{11}$.

4. Comenzando por la composición, los senadores elegidos serían 252 y, por tanto, un número reducido respecto al actual, a los cuales se añadirían dos representantes de cada Región y dos para cada provincia autónoma del Trentino Alto-Adige con un total de 42, elegidos, respectivamente, por el Consejo Regional y el Consejo de las autonomías locales. Ciertamente y como recoge el proyecto de ley, los 42 representantes de procedencia territorial "participan en la actividad del Senado federal sin derecho a voto, según las modalidades previstas por su Reglamento", pero en la composición compleja del Senado se van a agregar a los 252 y, por tanto, tendremos 294 senadores en total, por lo tanto, sólo 19 menos que en la actualidad. Omito examinar, limitándome exclusivamente a poner de manifiesto los problemas que podrán plantearse con referencia al status de los 42 representantes regionales en el Senado; esto es, deberían considerarse senadores a todos los efectos y, por tanto, gozar de todas las prerrogativas parlamentarias, así como participar en la actividad de designación, por ejemplo de los jueces constitucionales, o bien, podrán estar entre quienes componen la Asamblea de la República que elige al Presidente de la República. Por cuanto se refiere a los 252 senadores, que podremos denominar pleno iure, éstos serán elegidos en cada una de las Regiones contemporáneamente a la elección del respectivo Consejo regional. Esto es, significa que el Senado ya no será un órgano intermitente, con duración prefijada, como la Cámara, sino que más bien se convierte en un órgano continuo, cuyos componentes son elegidos simultáneamente con la elección de los Consejos regionales (o provinciales, en el caso de las Provincias autónomas) y permanecen en el cargo hasta la fecha de proclamación de los nuevos senadores de la misma Región o Provincia autónoma. De este modo, habrá no más una verdadera y propia elección del Senado, sino más bien la elección de "grupos" de senadores de una o varias Regiones. Este mecanismo de la simultaneidad electoral entre senadores y consejos regionales podrá también no convencer porque se considera débil para los fines de la creación de una au-

10 Consúltese, G. ZAGREBELSKY, Adeguamenti e cambiamenti della Costituzione, en Scritti in onore di Vezio Crisafulli, vol.II, Padova, Cedam, 1985, p.915 ss.

11 Entre los primeros comentarios al proyecto de Senado federal, puede consultarse M. MANETTI, Alcune riflessioni sul c.d. Senato federale, en la página web: www.associazionedeicostituzionalisti/dibattiti; Id., Il Senato federale all'italiana, en la página web: www.federalismi.it, n. 8, 2005; R. BIN, Senato federale? "... ma mi facci il piacere!", en la página web: www.forumcostituzionale.it; G. M. SALERNO, Brevi note sulla composizione e sull'organizzazione del Senato federale nel d.d.l. approvato, con modificazioni, dalla Camera dei deputati, y por último, también la página web: www.federalismi.it, n. 21, 2004. 
téntica representación territorial en el Senado; recordemos que resultaba absolutamente necesario encontrar un sistema que vinculase los senadores al territorio, a fin que se sintieran expresión del mismo,dado que la designación de los mismos por parte de los Gobiernos regionales -modelo Bundesrat alemán- era realmente muy difícil de lograr (si bien ésta parece ser la mejor solución). La elección simultánea es un método original, que deberá reforzarse con la praxis, que a veces resulta más eficaz que la norma escrita, y que podrá contribuir a hacer políticamente fuerte el nexo senadores-consejos regionales, en cuanto se vendrá a crear una relación de recíproca solidariedad y de apoyo político triunfante, que podrá inducir a hacer mayormente homogéneas las respectivas opiniones. A propósito de las elecciones coetáneas destacan aquí algunas incongruencias técnicas relativas a la aplicación correcta del mecanismo de la simultaneidad, en cuanto contrasta con la norma que sanciona el número fijo de senadores, 252, y la que impone el respecto de las proporciones entre los escaños y la población de cada Región. En la práctica, en un sistema en el cual los senadores cesan del cargo de "grupos regionales", en cuanto ligados al vencimiento de los respectivos Consejos regionales, no parece posible conciliar el número fijo de 252 senadores y la distribución proporcional escaño-población. Incorporo un ejemplo que aclara mejor esta cuestión: si se procede a la renovación del Consejo y de los respectivos senadores de la Región " $\mathrm{X}^{\text {" tras un }}$ censo de la población que haya registrado un aumento de población tal que da lugar a escaño posterior ¿qué sucede? Si se aumenta en uno los escaños correspondientes a la Región "X", habrá un Senado con 253 senadores vulnerando el artículo 57 que impone el número fijo de 252. Por el contrario, si no se aumenta un escaño a la delegación de los senadores, se vulnera la norma que impone la proporcionalidad entre escaño y población. En suma, un enigma...

Existen otros aspectos referidos a la futura composición del Senado federal, como la eliminación de los senadores vitalicios transferidos, por así decirlo, a la Cámara de los diputados, así pues la abolición de los senadores elegidos por la circunscripción externa. Después se ha aumentado ligeramente el número mínimo de senadores para cada Región, de cinco a seis; mientras que se ha disminuido el límite anagráfico para el electorado pasivo; por tanto, para ser elegido senador bastará tener 25 años. Por lo demás, queda sobre el escenario el problema de qué sistema electoral regirá para la elección de los senadores. En una primera versión, el proyecto de reforma constitucional tenía previsto y, por tanto, constitucionalizado, el sistema proporcional como fórmula electoral para la elección de los senadores. Esta referencia explícita ha sido oportunamente eliminada y, en consecuencia, corresponderá al legislador determinar el mecanismo electoral que deberá ser uno que "garantice la representación territorial por parte de los senadores". En fin, los senadores, como también los diputados, no representan sólo a la Nación, sino también a la República según la original novedad del artículo 67 de la Constitución.

5. A continuación pasaré a examinar las competencias del Estado federal, con particular referencia a los procedimientos legislativos. En primer lugar, debe afirmarse que el nuevo artículo 70 de la Constitución, tal y como se pre- 
senta en el proyecto de reforma, pone en marcha un procedimiento legislativo bastante complejo, cuyo único valor se encuentra en prever un inicio y un final y, por tanto, superar de tal modo la tradicional dificultad del actual procedimiento legislativo que, en la navette entre Cámara y Senado, puede no terminar nunca. Es indudable que leer el artículo al completo resulta pesado, fundamentalmente porque está mal redactado y es excesivamente largo y complicado en su desarrollo normativo. Se trata de un procedimiento legislativo "tripartito", por así decirlo, en cuanto se prevén tres categorías de leyes con tres iter legislativos diferentes: $a$ ) las leyes con preferencia en la Cámara; $b$ ) las leyes con preferencia en el Senado; $c$ ) las leyes bicamerales. La tripartición del procedimiento legislativo está determinada, de modo fundamental, por el contenido del proyecto de ley que es el parámetro para la elección del tipo de procedimiento a adoptar, así como para la preferencia o menos de una Cámara.

Intentemos sintetizar:

5.1. Las primeras, esto es, las leyes aprobadas con preferencia de la Cámara, son aquellas que la Cámara de los diputados examina en primer lugar y sobre las cuales tendrá la última palabra. El Senado puede proponer modificaciones en el plazo de 30 días, las cuales corresponderá a la Cámara decidir en vía definitiva. Se trata de leyes sobre todas las materias de competencia exclusiva estatal (del artículo 117 de la Constitución), salvo aquellas relativas a los denominados niveles esenciales, a los fundamentos sobre los entes locales y a los sistemas de elección de la Cámara y Senado, que, por el contrario, son de competencia bicameral.

5.2. Las segundas, esto es, las leyes con preferencia del Senado, son exactamente un reflejo de las que tienen preferencia en la Cámara, incluso como iter legislativo, con la excepción de que para éstas se prevé un eventual procedimiento de especial "confianza indirecta"; o bien, el Gobierno tiene la posibilidad de intervenir para hacer valer su propia convicción mediante un procedimiento especial, sobre un proyecto de ley que sería con preferencia del Senado. Se trata de un procedimiento complejo que puede, además, involucrar al Presidente de la República obligándole a tomar una decisión política que contrastaría con el papel de garantía que el propio proyecto de reforma constitucional le asigna. La intervención gubernamental que prevalece sobre el Senado puede ejercitarse "en positivo", esto es, con el fin de imponer al Senado, que no lo quiere, un texto que realiza el programa de Gobierno, $y$ «en negativo", por así decir, esto es, por contraste al Senado que quiere un texto considerado lesivo para los intereses unitarios vitales. Además, hay que decir que las leyes a prevalenza del Senado se refieren todas a materias de competencia concurrente (ex artículo 117 de la Constitución), aquellas que afectan a los principios fundamentales en las materias de competencia regional. Cierto, el problema, pues, es la definición, bastante controvertida, de que sean los uprincipios fundamentales"; en efecto, no hay en el ordenamiento y en la jurisprudencia constitucional una definición determinada y unívoca.

5.3. Por último, la tercera categoría de leyes, las bicamerales, son aquellas que requieren del concurso paritario de la Cámara y el Senado. Se trata de 25 ti- 
pos de leyes bicamerales que ahorro enumerar y remito a la no fácil lectura del artículo 70 apartado $3 .^{\circ}$ del proyecto de ley constitucional aprobado por el Parlamento, que no ha aclarado por qué obliga a un continuo reenvío a otros artículos de la Constitución, donde hay una expresa reserva de ley del Estado o de la República. Este procedimiento de las leyes bicamerales consiente al Senado, en algunos casos, desarrollar una significativa función de garantía, como por ejemplo para las leyes electorales o para la función fundamental de los Ayuntamientos, o bien, de coordinación en el caso por ejemplo de las redes de comunicación o de la investigación científica. Sin embargo, hay que decir que en otros casos el procedimiento bicameral interfiere - como para la determinación de los niveles esenciales de las prestaciones concernientes a los derechos civiles y sociales- en el ejercicio de una delicada función de Gobierno, que no puede sustraerse del circuito cuerpo electoral-Cámara de los Diputados-Gobierno.

Aún no hemos terminado. Existe una especie de posterior procedimiento legislativo, si bien excepcional. Es el que prevé cuando un proyecto de ley no fuese aprobado por ambas Cámaras, la intervención del Presidente de la Cámara y del Senado, quienes pueden convocar una comisión de 30 diputados y 30 senadores encargada de proponer un texto unificado para someterlo a votación final de ambas Cámaras. Además, hay que decir que la decisión de los Presidentes o de la comisión no es controlable en ninguna sede. Nos encontramos ante un punto jurídicamente delicado por las consecuencias que la exacta definición de su ámbito tiene en relación con la decisión sobre la cuestión de competencia y una eventual elevación de un juicio de constitucionalidad para los vicios de procedimiento.

Hay que referirse, además, al cómo afecta la figura y el papel de la Conferencia Estado-Regiones sobre la función legislativa del Senado federal, que ha sido constitucionalizada asignándola la tarea de srealizar la leal colaboración y promover acuerdos e entendimientos". En consecuencia, existe la posibilidad concreta de que sea este órgano, y no el Senado federal, el que desarrolle la función de mediación, determinando el contenido de las iniciativas legislativas y consecuentemente vaciando de significado la sede parlamentaria ${ }^{12}$. El riesgo de que se cree una especie de tricameralismo, por añadidura al conflicto, no aparece muy lejano...

6. A continuación haremos referencia, aunque sea rápidamente, a algunas cuestiones concernientes a las relaciones entre el Senado federal y el resto de órganos constitucionales.

6.1. Senado federal y Presidente de la República. Aparte de la elección de este último mediante la Asamblea de la República de la que forman parte los 252 senadores elegidos, pero no, a mi parecer, los 42 senadores de procedencia territorial, se observa como el Presidente del Senado, que continua siendo el Presidente de la República suplente, podría cesar del cargo de senador, en re-

12 Cfr. C. BASSU, La Conferenza Stato-Regioni nella riforma costituzionale, en el volumen: Cittadini, Governo, Autonomie. Quali riforme per la Costituzione?, dirigido por T. Groppi e P. L. Petrillo, Milano, Giuffrè, 2005, p.141 ss. 
lación a los cambios del Consejo de las Regiones de referencia; también, durante el ejercicio de las funciones delegadas del Presidente de la República, y éstas debían ser asumidas por el vicepresidente suplente del Senado. Así pues, será una hipótesis de laboratorio, pero resulta necesario destacarla también por su extravagancia.

6.2. Senado federal y Gobierno. Ya he afirmado con relación al procedimiento legislativo y a la forma de "confianza indirecta", y destaco de nuevo aquí, el hecho de que no exista ningún vínculo fiduciario entre el Gobierno y el Senado federal, y correlativamente la posibilidad de disolución de este último. Efectivamente, el Primer Ministro ilustra el programa de legislatura y la composición del Gobierno entre las Cámaras y, por tanto, también en el Senado, pero sólo la Cámara de los diputados se pronuncia con un voto sobre el programa. En resumidas cuentas, por cuanto se refiere al control no está claro si éste puede ejercitarse por parte del Senado federal también sobre materias exteriores y concurrentes, sobre las que el Senado es competente para su primera lectura.

6.3. Consejo Superior de la Magistratura, Corte Constitucional y Senado federal. Este último interviene en el momento de elección de una parte de los componentes de ambos órganos. Concretamente, por lo que se refiere al Consejo Superior de la Magistratura, el Senado elige una sexta parte de los componentes (el mismo número es elegido por la Cámara de los diputados); por cuanto concierne a la Corte Constitucional, el Senado procede a la elección de cuatro magistrados (en cambio, tres los elige la Cámara) y lo hace en una composición de la Asamblea renovada con la integración de los Presidentes de las Juntas regionales y de las Provincias autónomas de Trento y Bolzano. Resulta complicado comprender las razones de una novedad como esta.

7. Ahora procederé a desarrollar algunas consideraciones finales. Sin embargo, antes quisiera subrayar un aspecto que no se ha sobrevalorado, el referido a las disposiciones transitorias en el proyecto de ley de reforma constitucional. En efecto, se trata de cinco artículos, algunos con diversos párrafos, de difícil comprensión. Piénsese sólo en la posibilidad de la entrada en vigor de la reforma constitucional que puede estratificarse temporalmente en varias fases. Y después, con particular referencia a la elección de senadores federales, que deberían calibrarse con las elecciones de los respectivos Consejos regionales. Un escenario que podría tenerse sería el siguiente: en 2011 se celebrarán las elecciones para la primera legislatura del Senado federal compuesto aún por 315 miembros, y los consejos regionales, elegidos en 2010, en el cargo más o menos hasta finales de 2013, serán prorrogados hasta 2016. Será este año en el que comience el nuevo Senado federal compuesto por 252 miembros elegidos contemporáneamente a los Consejos regionales. En suma, si todo va bien, el Senado federal de la República lo tendremos dentro de once años...

Debo decir que esta fecha de entrada en vigor del Senado federal tan lejana, termina atenuando y disminuyendo las críticas que podrían presentarse al proyecto. En efecto, parece dificilísimo imaginar que todo pueda interrumpirse y no se modifique nada hasta 2016, año de inicio del nuevo Senado federal. 
Resulta más fácil prever más bien cambios posteriores y consideraciones sustanciales (o desaprobaciones en el caso de un referéndum afirmativo), tales para alterar el proyecto hoy en discusión.

Dicho esto, me limitaré a desarrollar una consideración de máxima, que es la siguiente. Por su composición y funciones, el Senado federal parece configurarse como un órgano político especializado en el tratamiento de cuestiones regionales. Por tanto, el bicameralismo que se querría crear, sería en realidad un bicameralismo por especialización o funcional, más que un bicameralismo con representación diferenciada. Lo que podría no ser un problema. Pero aquí hay que señalar la incongruencia que se anida en la parte relativa a las competencias del Senado federal; especialmente, porque éstas se referirían a toda la legislación estatal concurrente del artículo 117 de la Constitución. Teniendo en cuenta que el Senado no debiera ser una Cámara política, asignándola esta misma competencia, se corre el riesgo de crear un doble monocameralismo, en lugar del actual bicameralismo paritario. En efecto, de una parte, se excluye al Senado de la relación fiduciaria con el Gobierno, mientras que, de otra, se le confía la última y decisiva palabra sobre las leyes relativas a la determinación de los principios fundamentales en las materias de legislación concurrente. Una competencia ésta, que sería más oportuno asignarla a la Cámara, desde el momento que se trata de materias relativas a las políticas de sectores significativos para el indirizzo politico del Gobierno. Imagínese un Senado federal como órgano de contrapeso institucional de la mayoría, inspirándose así en la reciente tesis de Bruce Ackerman sobre la nueva separación de poderes ${ }^{13}$, que viene a auspiciar continuas y repetidas formas de conflicto, que perjudicarían gravemente el parlamentarismo y la gubernabilidad.

Resulta incuestionable que la concreta adaptación del Senado al orden constitucional será también, y sobre todo, confiada al principio de efectividad, a su desarrollo en la Constitución en sentido material. Sólo de este modo, el Senado y los senadores podrán conquistar un rol previsto por el ordenamiento, eliminada la angustia de convertirse en los «inválidos de la Constitución.

ABSTRACT. On November 16th, 2005 the Italian Parliament passed a strongly relevant modification of the second part of the Constitution. One of the most important innovations is represented by the change of the Senate which is supposed to become a "federal" one and because of that, it is meant to be representative of Regions. This is the end of the perfect and equiordinated bicameralism which has always been a typical characteristic of the Italian Bica-

13 Sobre estas cuestiones véase, B. ACKERMAN, La nuova separazione dei poteri, tr. it., Roma, Carocci, 2003; sobre este trabajo, puede consultarse también mi recensión crítica publicada en Diritto Pubblico Comparato ed Europeo, n. 2, 2003. 
meralism. The reform of the Senate comes consequently to the modification of the 2001 reform of "Titolo V" of Italian Constitution, which contemplated the division of competences between State and Regions. In the light of this came the exigence of creating forms of regional representation within one of the two Houses of Parliament, in order to permit the partecipation to the elaboration of the legislative process concerning territorial autonomies, and avoiding in this way conflicts of attribution between State and Regions, relating to the prerogatives of territorial entities. Of course, it is sure that formal change has to be followed by the realization of a new bicameralism good to satisfy the necessities of Italian federal system. The reform of the Senate does not seem to fully satisfy such a necessity. In particular, the new legislative process is quite complex and hard to realize. It is a "triple" legislative proceeding, because there are three different categories of Acts with three different Legislative iters: a) Acts with a prevalence of the House of Deputees; b) Acts with a prevalence of the Senate; c) Bicameral Acts. The distinction in three ways to the Legislative process is determined, in its substance, by the content of the draft of the Act, which is the parameter used for choosing the kind of proceeding that will be adopted (and it is the criterion for distinguishing if it is the House or the Senate to prevail). Moreover, we have to say that because of its composition and of its functions, the Senate looks more like a specialized political organism dealing with regional affairs. The kind of bicameralism that it is supposed to give birth to, would actually be characterized by specialization or by functional distinction more than an organism created useful to guarantee a differentiated representation. 